\title{
Combining Optical Coherence Tomography with Fluorescence Imaging
}

\author{
Shuai Yuan and Yu Chen \\ Fischell Department of Bioengineering, University of Maryland \\ College Park, Maryland 20742, \\ U.S.A.
}

\section{Introduction}

\subsection{Overview of optical coherence tomography}

Optical coherence tomography (OCT) is a promising imaging technology which can provide subsurface imaging of biological tissues (Huang et al., 1991). OCT images are similar to the ultrasound images, but with significantly higher resolution (5-10 times) than the clinical ultrasound. OCT enables imaging of tissue microstructure with micron-level resolution, approaching that of standard excisional biopsy and histopathology, except that imaging can be performed in real time without the removal of a tissue specimen.

The optical configuration of OCT is the same as that of a low coherence interferometer (LCI). Low-coherence interferometry is a type of optical interferometry based on low-coherent light sources (Mandel \& Wolf, 1995). This technique is capable of measuring depth-resolved (axial, z) tissue structure, birefringence, flow (Doppler shift), and spectra at a micrometerlevel resolution by precisely measuring the amplitude and the relative phase of reflected or backscattered light. Optical interferometer has been invented for 120 years. But most of the time, it was just laboratory equipments for physics research until 1970s. In the early 1980s the emergence of high brightness semiconductor broadband light sources and single mode optical fibers stimulated the development of LCI. The availability of superluminescent diodes (SLD) made low-coherence reflectometry practical in a fiber-optic system (Wang et al., 1982). SLD could be used in LCI to achieve a distance resolution of about $10 \mu \mathrm{m}$. Another innovation was the development of fiber optic devices such as couplers which are important in the development of fiber optic based designs. These technological developments are the prerequisites of current OCT system used for noninvasive optical imaging of living tissues in vivo.

In 1980s, the LCI technique was mainly developed by several groups for reflection measurements in telecommunications devices with micron resolution (Danielson \& Whittenberg, 1987; Youngquist et al., 1987). The potential of LCI for three-dimensional imaging in biological tissue was first realized in 1991 (Huang et al., 1991). Since the original work, a large number of papers have been published regarding various aspects of OCT. OCT has been successfully translated to various clinical applications including ophthalmology (Schuman et al., 2004), cardiology (Jang et al., 2005), gastroenterology (Bouma et al., 2000; Li et al., 2000; Sivak et al., 2000; Chen et al., 2007a), dermatology (Welzel 
et al., 2004), dentistry (Otis et al., 2000), urology (D'Amico et al., 2000), among others (Huang et al., 1991; Fujimoto et al., 1995; Brezinski et al., 1996; Tearney et al., 1997; Fujimoto et al., 2000; Fujimoto, 2003).

Figure 1A shows a schematic of the time-domain OCT (TD-OCT) system. Measurements are performed using a Michelson interferometer with a low coherence length light source. One arm of the interferometer directs the light onto the sample and collects the backscattered signal. The other interferometer arm generates a reference signal. Optical interference between the light from the sample and reference mirror occurs only when the optical distances traveled by the light in both the sample and reference paths match to within the coherence length of the light. Low coherence interferometry permits the optical path length and magnitude of the light reflected from tissue microstructures to be measured with extremely high accuracy and sensitivity. Cross-sectional images are constructed by laterally scanning the optical beam and performing sequential axial measurements of backscattered light at different transverse positions (see Figure 1B). Three-dimensional (3D) volumetric images can be obtained similarly by steering the beam in two dimensions.
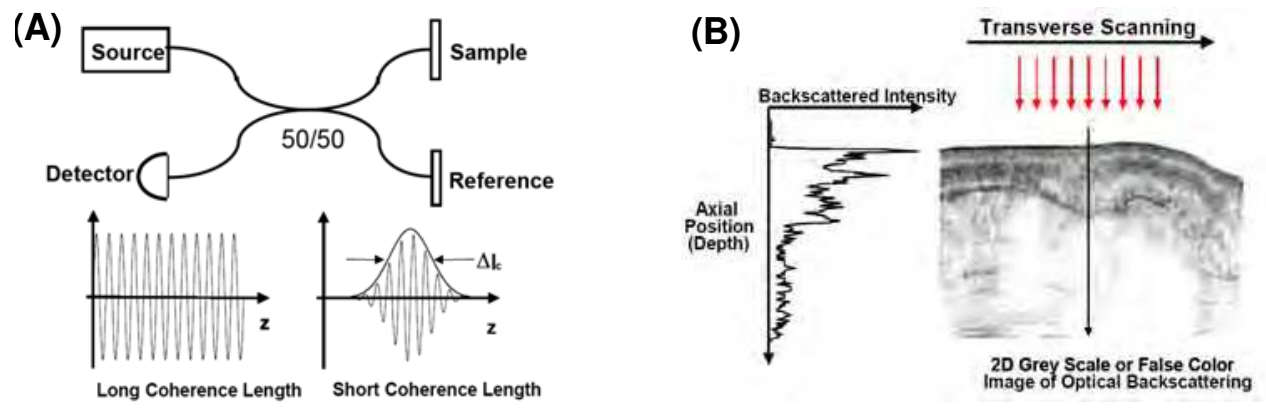

Fig. 1. (A) Optical Coherence Tomography (OCT) generates images by measuring the echo time delay of light. The system uses a fiber optical Michelson interferometer with a low coherence light source. (B) Cross-sectional images are generated by scanning a beam across the tissue and measuring the backscattering intensity as a function of depth. A gray scale or false color image can then be displayed.

Although time domain OCT is successful in many biomedical applications, it is hampered in achieving real-time imaging mainly by the relatively complicated mechanical designs for dual scanning, a mechanical delay line scanning in the reference arm for coherence gating and a position scanning in the sample arm. An alternate approach to coherence gating that does not employ a scanning delay line is to acquire a broad band spectrum of the backscattered interferometric signal generated by mixing sample light with reference light at a fixed group delay and then obtain depth-scan information by an inverse FT of the spectrum. This approach is called Fourier domain OCT (FD-OCT). Two distinct methods have been developed that employ this FD-OCT approach. The first, spectral domain OCT (SD-OCT) (Fercher et al., 1995; Wojtkowski et al., 2002) uses a broadband light source and achieves spectral discrimination with a dispersive spectrometer in the detector arm. The second method, swept source OCT (SS-OCT) (Fercher et al., 1995; Chinn et al., 1997; Haberland et al., 1998) achieves spectral discrimination by rapidly tuning a narrowband source through a broad band in the source arm. Schematics of a SD-OCT system and a SSOCT system are shown in Figure 2. FD-OCT system can not only achieve a fast frame 
acquisition rate as it is designed for, but it can also achieve superior sensitivity (defined as the signal-to-noise ratio (SNR) for a perfect sample reflector) over TD-OCT (Huber et al., 2006). Recently, FD-OCT becomes more and more popular in biomedical research. Commercialized FD-OCT systems have been in the market for several years and were used widely in many recent studies. In this chapter, all of our newly developed multi-modality systems are based on SS-OCT.
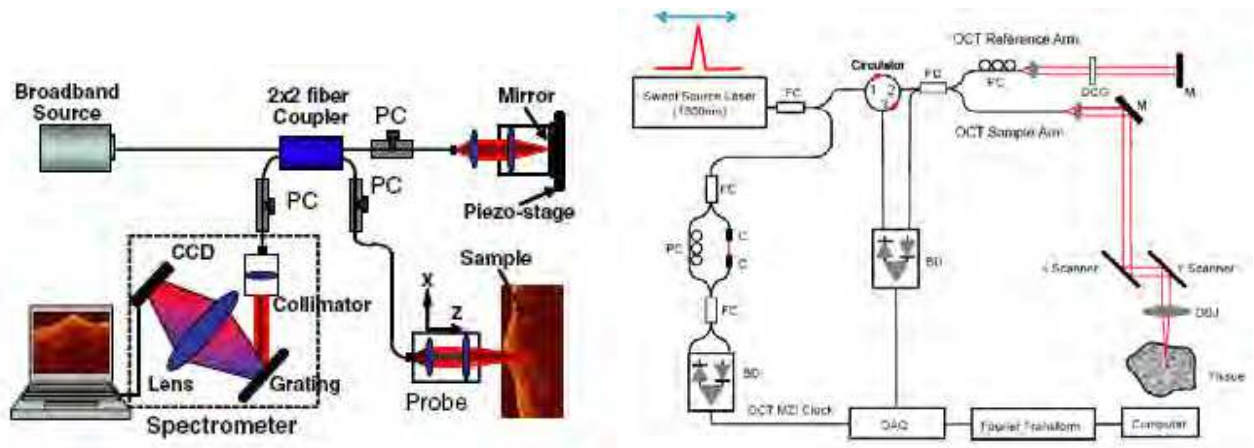

Fig. 2. Typical SD-OCT (left) from (Wang, 2007) with permission and SS-OCT system (right).

\subsection{Principles of Swept Source Optical Coherence Tomography (SS-OCT)}

Detailed aspects of traditional time-domain OCT theory and applications, such as resolution, sensitivity, noise, signal-to-noise ratio, etc, could be found in many literatures (Schmitt, 1999; Fercher et al., 2003), and will not be covered in this chapter. For FD-OCT, the optical paths of light beam are the same as those in TD-OCT techniques. The light beam from the source is split by a fiber coupler or beam splitter and comes through and back in the reference arm and the sample arm. The beam is then recombined at the coupler or the beam splitter and forms an interferometric signal. In SD-OCT a spectrogram is obtained by using a dispersive spectrometer with a CCD camera in the detector arm, and in SS-OCT a spectrogram is obtained by using a frequency swept laser or tunable laser with just a single detector and without dispersion components.

In SS-OCT, the spectrum of the light source is described as a discrete spectral intensity distribution with a line width $\sigma_{k}$ and tuning mode-hop $\boldsymbol{\delta}_{k}{ }^{\prime}$. The interferometric signal at the detector can be described as (Liu \& Brezinski, 2007):

$$
\begin{aligned}
I_{D}(k)= & G(k)\left[e^{-k^{2} / 2 \sigma_{k}^{2}} \otimes \operatorname{comb}\left(k / \delta_{k}{ }^{\prime}\right)\right]\left[p_{R}^{2}+\int_{0}^{z} p_{S}^{2}\left(z^{\prime}\right) d z^{\prime}+\right. \\
& \left.2 \operatorname{Re}\left\{\int_{0}^{z} p_{S}\left(z^{\prime}\right) p_{R} e^{j 4 \pi k\left[n_{s}\left(k_{0}\right) z^{\prime}-z_{R}\right]} d z^{\prime}\right\}\right]
\end{aligned}
$$

where $k$ is the wavenumber, $I_{D}(k)$ is the detected light intensity at wavenumber $k, G(k)$ is the spectral coefficient combing the intensity spectrum of the light beam and the conversion/coupling factors from the source to the detectors, $p_{R}$ represents the fixed backscattering coefficient in the reference arm, $p_{S}(z)$ represents the backscattering coefficient at the position $z$ in the sample, and $n_{S}(k)$ is the refractive index of the sample at wavenumber $k$. Both $p_{R}$ and $p_{S}(z)$ include coupling factors from the fiber coupler or beam 
splitter. The depth variable $Z^{\prime}$ is measured from $\boldsymbol{O}_{s}$, the origin of coordinates in the sample arm. Consequently the origin of coordinates in the reference $\operatorname{arm} \boldsymbol{O}_{R}$ is the point from where the optical group delay to the coupler matches that between $O s$ and the coupler in the sample arm. The mirror position is $Z_{R}$ measured off the $\boldsymbol{O}_{R}$. In Eq. (1), the first term within the second square brackets is the signal from the reference arm; the second term comes from the sample arm and encodes the depth information of the object; the third term described the mutual interference of all scattering waves.

The Fourier transfer of Eq. (1) can be expressed as (Liu \& Brezinski, 2007):

$$
\begin{aligned}
F^{-1}\left\{I_{D}(k)\right\}= & F^{-1}[G(k)] \otimes\left[e^{-2 \sigma_{k}^{2} z^{\prime 2}} \operatorname{comb}\left(\delta_{k}{ }^{\prime} z^{\prime \prime}\right)\right] \otimes \\
& {\left[F^{-1}\left\{p_{R}^{2}+\int_{0}^{z} p_{S}^{2}\left(z^{\prime}\right) d z^{\prime}\right\}+p\left(\frac{z^{\prime \prime}+2 Z_{R}}{2 n_{s}\left(k_{0}\right)}\right)+p\left(\frac{-z^{\prime \prime}+2 Z_{R}}{2 n_{s}\left(k_{0}\right)}\right)\right] }
\end{aligned}
$$

From Eq. (2), it is seen that the mode-hop $\boldsymbol{\delta}_{k}{ }^{\prime}$ will limit the $A$-scan range and the laser line width $\sigma_{k}$ will degrade the $A$-scan profile.

In SS-OCT, the sensitivity improvement is obtained through higher spectral intensity of the laser source. In a swept or tunable laser source, the intensity over a small range of the spectrum can be easily kept much higher than that of a wideband light source. Thus possible SNR improvement can be gained in SS-OCT (Huber et al., 2006). Furthermore, a bandpass filter technique can be used, by shifting the region of interest (ROI) of the sample fairly far off the plane $O_{S}$, to keep all the high frequency spectral oscillation introduced by the interested scatters and filter out much of the low frequency noise (Liu \& Brezinski, 2007). Another theoretical advantage of SS-OCT is that the tuning speed of the swept source could be high enough to shift the spectrum of the temporal spectral interferogram higher and out of the low frequency noise range, which is from zero to tens of kilohertz.

\section{Combining OCT with fluorescence}

Early detection of neoplastic changes before metastasis occurs remains a critical objective in clinical cancer diagnosis and treatment. Excisional biopsy and histopathology is currently the gold standard for cancer diagnosis. However, it can suffer from false negative rates due to sampling errors. Optical imaging technologies can provide real-time imaging of human tissues in vivo with resolutions approaching that of histopathology and have the potential to reveal the biochemical and/or molecular information; therefore they could significantly improve the identification of malignancies at curable stages. The ability to assess tissue architectural morphology (such as the alterations in glandular or stromal morphology) and molecular information (such as up-regulation of receptors and over-expression of enzymes), in vivo and in real time, without the need for tissue excision, would be a major advance in cancer diagnostics and therapy. There is a critical need to develop such an imaging technology with high sensitivity and specificity and with strong translational potential to clinical medicine.

Since OCT provides high-resolution, cross-sectional imaging of tissue microstructure in situ and in real-time, and fluorescence imaging provides the biochemical and metabolism information, there are great interests to combine these two modalities to provide the structural and functional information of the tissues in order to enhance the disease detection capability. Pan et al. suggested that ALA fluorescence-guided endoscopic OCT could enhance the efficiency and sensitivity of early bladder cancer diagnosis (Pan et al., 2003). In 
an animal model studies, they demonstrated that the specificity of fluorescence detection of transitional cell carcinoma was significantly enhanced by fluorescence-guided OCT ( $53 \%$ vs. $93 \%)$, and the sensitivity of fluorescence detection also improved by combination with OCT (79\% vs. 100\%) (Wang et al., 2005).

Tumlinson et al. have developed a combined OCT and laser-induced fluorescence (LIF) spectroscopy imaging catheter for in vivo mouse colon imaging (Tumlinson et al., 2004; McNally et al., 2006), as shown in Figure 3. The source beam from SLD is passed to a 50:50 fiber beam splitter and separated to the reference beam and the sample beam. The reference beam travels, through a neutral-density (ND) filter to a collimator, and then returns to the beam splitter after reflection from a retroreflector. The sample beam is coupled into a dualmodality endoscope. The light from the reference and sample paths is recombined at the beam splitter and travels to a detector. The LIF He-Cd laser source is coupled into a multimode fiber and into the dual-modality endoscope, too. Two adjacent collection fibers in the endoscope collect the emission light and carry it to the CCD spectrometer for analysis.

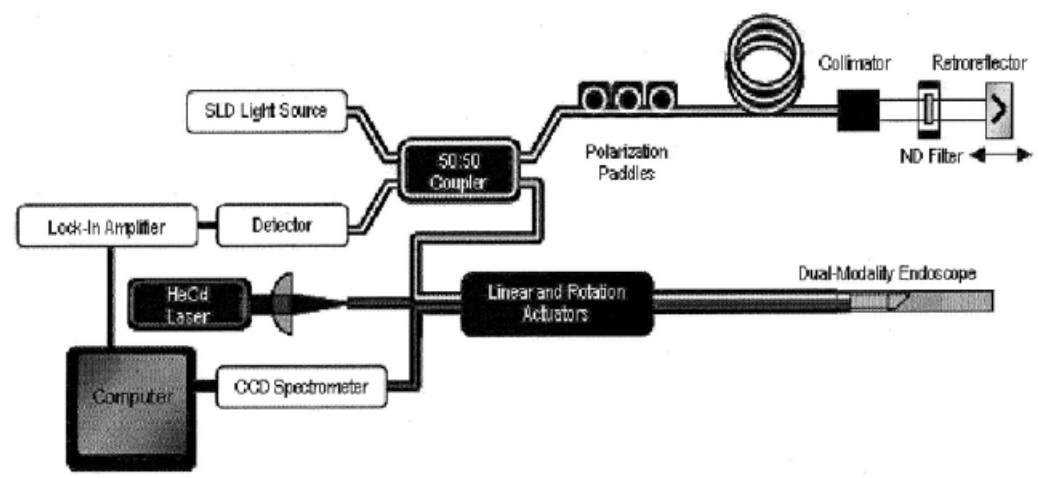

Fig. 3. Schematic of the OCT-LIF system (McNally et al., 2006) with permission.

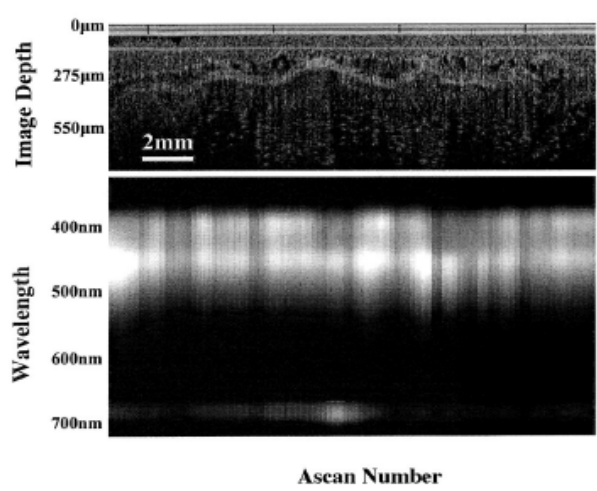

(A)

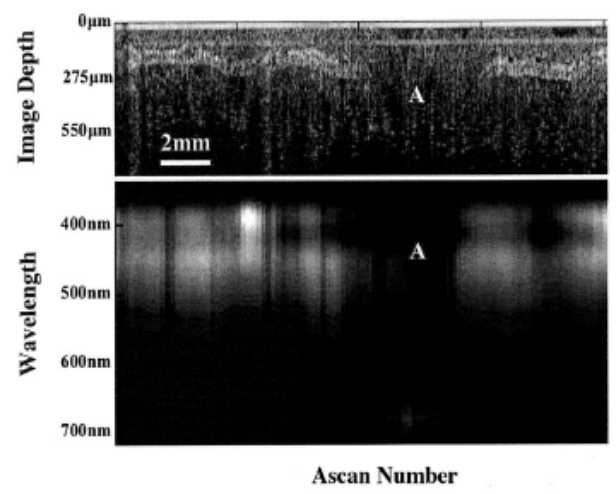

(B)

Fig. 4. OCT images (top) and corresponding LIF spectra (bottom) of healthy colon taken from a high-chlorophyll diet mouse (A) and adenoma (B) (McNally et al., 2006) with permission. 
This miniaturized $2 \mathrm{~mm}$ diameter catheter-based dual-modality system has been used to monitor the disease progression in mouse colon longitudinally, and is able to identify colorectal adenomas in murine models (Hariri et al., 2006; McNally et al., 2006; Hariri et al., 2007). In one study, McNally et al. used the OCT-LIF system to study healthy and adenomatous mouse colon. Their study found close agreement among each of the modalities and with histology. Both modalities were capable of identifying diseased tissue accurately. Here, the OCT images provided structural information about the tissue, while the autofluorescence signal measured by LIF provided biochemical information. Using this multi-modal system, they found that the magnitude of the $680 \mathrm{~nm}$ signal correlates with chlorophyll content in the mouse diet, suggesting that the autofluorescent compound is a dietary metabolite. Figure 4 shows their OCT image (top) and corresponding LIF spectra (bottom) of healthy colon taken from a high-chlorophyll diet mouse (left) and adenoma mouse (right). The LIF emission peaks at approximately 390 and $450 \mathrm{~nm}$, with an absorption dip at $420 \mathrm{~nm}$ (typical of healthy tissue). The presence of a distinct peak at approximately $680 \mathrm{~nm}$ is visible throughout the colon as well. The adenoma can be visualized in the OCT image (Region A in Figure 4B). The corresponding LIF signal shows a significant reduction in the 390 and $450 \mathrm{~nm}$ signals and a peak in the $680 \mathrm{~nm}$ signal over the adenomatous region.

\section{Combining OCT with Fluorescence Molecular Imaging (FMI)}

\subsection{Introduction}

Fluorescence molecular imaging (FMI) could obtain molecular information by measuring fluorescence intensity of fluorescent bio-markers which target specific molecules. Previous researches have shown that cancerous tumors can be identified with fluorescent markers (Tung et al., 2000; Achilefu, 2004). In a study done in mice, it was shown that adenomatous polyps in the colon express $36 \%$ more of the proteolytic enzyme cathespin B than normal tissue (Marten et al., 2002). Also, it was shown that mice colons that expressed more cathespin B, when injected with a fluorescent dye, showed significantly higher fluorescent intensities than mice injected with dye that did not have adenomatous polyps.

Although LIF technique and 2-D FMI technique have the same scientific bases and the silimiar abilities in monitoring molecular processes, FMI technique is more convenient in obtaining 2-D images showing flurophore's spatial distribution and molecular process spatial hetergenity using specifically-targeted dye. In our recent study, we combined the OCT with FMI system to investigate the correlation between OCT structural features and FMI molecular information. The system demonstrated that it could co-register en face OCT and FMI images. Relationships of FMI intensity and dye concentration as well as FMI intensity and target fluorescence tube depth were studied. The capability of imaging biological tissue was demonstrated by imaging the mouse colon cancer model ex vivo.

\subsection{Methods}

\subsubsection{Optical Coherence Tomography (OCT)}

Figure 5 shows the schematic of the co-registered OCT and fluorescence imaging system. The fiber-based high-speed, high-resolution OCT system utilizes the wavelength-swept laser as the light source. It generates a broadband spectrum of $100 \mathrm{~nm}$ at $1310 \mathrm{~nm}$, which provides an axial resolution of $10 \mu \mathrm{m}$ in the tissue. The laser operates at a sweep rate of 16 $\mathrm{kHz}$ (equivalent to an imaging speed of 30 frames per second for a 512 axial-line image) with an average output power of $12 \mathrm{~mW}$. The system sensitivity is $95 \mathrm{~dB}$. A Michelson 
interferometer composed of one circulator and a fiberoptic 50/50 splitter is used to generate the Fourier-domain OCT signal. The reference arm consists a stationary mirror $(\mathrm{M})$ and a polarization controller (PC). The light from the sample arm passes through a collimator (C) and is steered by a pair of galvanometer mirrors (X and $\mathrm{Y}$ ) then focused by an objective lens to a spot. The power on the sample is $4 \mathrm{~mW}$ with a spot size of $15 \mu \mathrm{m}$. The OCT interference signal returned from both the sample and reference arms is detected by a balanced photodetector (BD). A Mach-Zehnder interferometer (MZI) with a fixed path difference is used to generate an optical frequency clock. Data acquisition is triggered by the zerocrossings of the MZI fringes, which are evenly spaced in optical frequency. Discrete Fourier transform (DFT) is performed on the data to generate an axial depth profile of the sample (A-line) with $3 \mathrm{~mm}$ imaging depth and 512 pixels (Andrews et al., 2008).

\subsubsection{Fluorescence Molecular Imaging (FMI)}

The fluorescence molecular imaging (FMI) system uses continuous-wave (CW) laser diodes at $675 \mathrm{~nm}$ as the excitation source. The excitation light is combined with the OCT sample arm using a dichroic mirror. The typical illumination power on the sample is approximately $1 \mathrm{~mW}$. The reflectance and fluorescence light is detected by the same fiber and then connected to a fiber splitter to divide the collected light into reflectance and fluorescence signals. The simultaneous measurement of reflectance and fluorescence signals from the same source and detector geometry is important for minimizing the influence of optical coupling variation for both excitation and collection paths. The reflectance signals are detected by an avalanche photodiodes (APD), while the fluorescence signals first pass through an emission filter set $(700 \pm 10 \mathrm{~nm})$, and then are detected by a photomultiplier tube (PMT). The illumination and filter wavelength are chosen based on the excitation and emission properties of the near-infrared dye Cy5.5. Such a system can be readily adapted to image other fluorescence dyes with different excitation/emission wavelengths.

\subsubsection{Molecular imaging contrast agent}

Colorectal cancer is the third most common form of cancer, and the second leading cause of cancer death in the United States (Jemal et al., 2008). Recently, Roney et al. demonstrated that the legume lectin Ulex europaeus agglutinin I (UEA-1) binds the surfaces of adenomatous polyps in specimens of colorectal cancer from the APCMin mouse model. The carbohydrate a-L-fucose, which is over-expressed on the surfaces of polyps, facilitates the bond with UEA-1. Thus, a-L-fucose may be a possible biomarker to target and detect colon adenomas by molecular imaging methods (Roney et al., 2008).

The details of the synthesis of the molecular contrast agent UEA-1 conjugated polymerized liposomes have been described elsewhere (Roney et al., 2008). Basically, the contrast agent contains the fluorescence dye Lissamine Rhodamine PE, therefore, in order to imaging this specific contrast agent, we use a laser source with $532 \mathrm{~nm}$ excitation and a fluorescence filter with $600 \pm 10 \mathrm{~nm}$.

\subsubsection{Animal model of colon cancer}

Excised small and large bowels $(\mathrm{N}=4)$ of male C57BL/6J APCMin/+ mice were commercially purchased from the Jackson Laboratory (stock \#002020, $8 \mathrm{wks}$ ), and stored at $-80{ }^{\circ} \mathrm{C}$ until further use. The bowels were thawed to room temperature (RT) for 15-20 mins, cut along the longitudinal axis, and preserved in formaldehyde (RT, 15 mins) before the commencement 
of staining. The specimens were incubated in UEA-1 conjugated and non-conjugated liposomes (containing $0.4 \mu \mathrm{M}$ Lissamine Rhodamine PE) at RT for 45 mins. The specimens were then washed in PBS, before being mounted and imaged by OCT/FMI system.

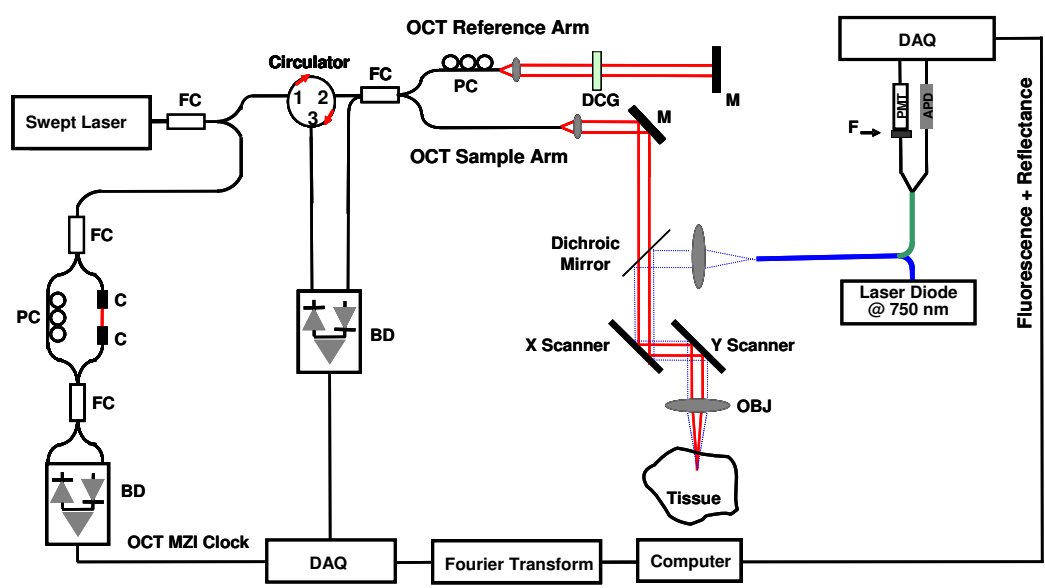

Fig. 5. Schematic of integrated OCT/FMI system. The left side is the OCT imaging subsystem and the right side is the FMI imaging subsystem. They are combined by a dichroic mirror in the sample arm. FC: fiber coupler; PC: polarization control; C: collimator, MZI: Mach-Zehnder interferometer (frequency clocks), M: mirror, BD: balanced detector, DAQ: data acquisition board, DCG: dispersion compensating glasses, OBJ: objective; PMT: photomultiplier tube; APD: avalanche photodiode; F: fluorescence filter (Yuan et al., 2010b) with permission.

\subsection{Characterization of FMI and OCT signal}

\subsubsection{Characterization of tissue scattering coefficients}

In a typical OCT A-scan, OCT signal I(z) decays with the depth z. Since 1990s, several theoretical models of OCT have been developed to fit OCT signals as a function of depth. Those models can be used to find the scattering coefficient $\mu_{\mathrm{s}}$ of the scattering media (Schmitt et al., 1993; Faber et al., 2004; Levitz et al., 2004; Turchin et al., 2005). Most recent models (Levitz et al., 2004; Turchin et al., 2005a) consider factors from the multi-scattering and the Gaussian beam propagation. They can produce a very good fit on A-scan profile as well as a close match of the scattering coefficient value to the MIE theory. In our study, we used the model from Letivz et al. (2004) to fit our experimental data and then find the scattering coefficient:

$$
I_{t}(z)=I_{0}\left\{e^{-2 \mu_{s} z}+\frac{4 e^{-\mu_{s} z}\left[1-e^{-\mu_{s} z}\right]}{1+\frac{w_{S}{ }^{2}}{w_{H}{ }^{2}}}+\left[1-e^{-\mu_{s} z}\right]^{2} \frac{w_{H}{ }^{2}}{w_{S}{ }^{2}}\right\}
$$

The first term in Eq. (3) represents the single scattering contribution, the third term represents the multiple scattering contribution, while the second term is the cross-term. $\mathrm{w}_{\mathrm{H}}$ and $w_{S}$ reflects contribution from the Gaussian beam propagation. $w_{H}, w_{S}$ are the 1/e Gaussian beam irradiance radius at the probing depth in the absence of scattering and in the presence of scattering, respectively. They can be defined as: 


$$
\begin{gathered}
w_{H}^{2}=w_{0}^{2}\left(A-\frac{B}{f}\right)^{2}+\left(\frac{B \lambda}{2 \pi w_{0}}\right)^{2} \\
w_{S}^{2}=w_{0}^{2}\left(A-\frac{B}{f}\right)^{2}+\left(\frac{B \lambda}{2 \pi w_{0}}\right)^{2}+\left[\frac{B \lambda}{\pi \rho(z)}\right]^{2} \\
\rho(z)=\sqrt{\frac{3}{\mu_{S} z}} \frac{\lambda}{\pi \theta_{r m s}}\left(\frac{n B}{z}\right)
\end{gathered}
$$

Here $\rho(z)$ is the lateral coherence length. A and B are elements from the ABCD ray-matrix for light propagation from the lens plane to the probing depth in the sample. If the focal plane of the sample beam is fixed on the surface of the sample, $A=1$ and $B=d+z / n$. $d$ is the distance between the lens and the surface. $w_{0}$ is the 1 /e irradiance radius of the input sample beam at the lens plane, $\lambda$ is the center wavelength of the source, $\mathrm{f}$ is the focal length of the lens, $\mathrm{n}$ is the mean index of refraction of the sample and $\theta_{\mathrm{rms}}$ is the root-mean-square scattering angle. We could find the value of scattering coefficient $\mu_{\mathrm{s}}$ by minimizing the mean-square deviation of the logarithms of the experimental curve $\mathrm{I}_{\mathrm{e}}(\mathrm{z})$ and fitting theoretical curve $\mathrm{I}_{\mathrm{t}}(\mathrm{z})$ :

$$
\left[\mu_{s}, \theta_{r m s}\right]=\arg \min _{\left(\mu_{s}, \theta_{m s s}\right)} \sum_{n} \log ^{2}\left[I_{t}\left(z_{n}\right) / I_{e}\left(z_{n}\right)\right]
$$

We have used this method to find scattering coefficients of polystyrene microspheres and intralipid phantom. The obtained values are close to the values estimated by MIE theory or values reported in literatures (Vanstaveren et al., 1991; Flock et al., 1992; Zaccanti et al., 2003), as seen in Table 1 and Figure 6. For comparison, fitting result from Faber's model is also presented in Table 1 (Faber et al., 2004). This model, as shown in Eq. (8), includes single scattering term in Eq (3) and a factor for axial point spread function. The model works better for weakly scattered tissues.

$$
I_{t}(z)=I_{0} \frac{1}{\left(\frac{z-z_{c f}}{z_{R}}\right)^{2}+1} e^{-2 \mu_{s} z}
$$

Here $Z_{c f}$ is the position of the confocal gate and $Z_{R}$ is the apparent Rayleigh length.

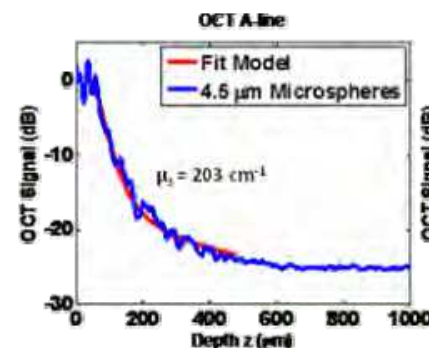

(A)

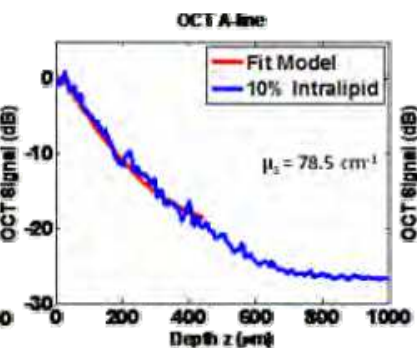

(B)

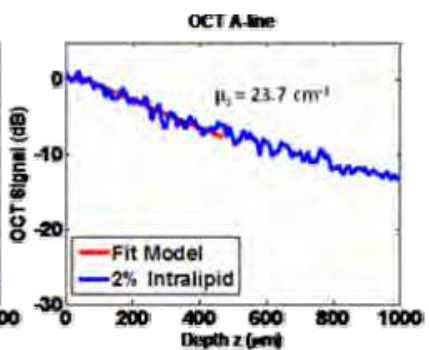

(C)

Fig. 6. OCT A-scan profiles and their fit result to the model Eq. (3). 6A is on microsphere phantom ( $4.5 \mu \mathrm{m}$, Polysciences); $6 \mathrm{~B}$ is on $10 \%$ intralipid solution; $6 \mathrm{C}$ is on $2 \%$ intralipid. 


\begin{tabular}{|c|c|c|c|}
\hline Phantom & $\begin{array}{c}\mu_{\mathrm{s}}\left(\mathrm{cm}^{-1}\right) \text { from } \\
\text { fitting Eq. (3) }\end{array}$ & $\begin{array}{c}\mu_{\mathrm{s}}\left(\mathrm{cm}^{-1}\right) \text { from } \\
\text { fitting Eq. }(8)\end{array}$ & $\begin{array}{c}\mu_{\mathrm{s}}\left(\mathrm{cm}^{-1}\right) \text { from } \\
\text { MIE or reported }\end{array}$ \\
\hline $4.5 \mu \mathrm{m}$ microsphere & 203 & 160.1 & $211^{\mathrm{a}}$ \\
\hline $10 \%$ Intralipid & 78.5 & 69.7 & $63-82^{\mathrm{b}}$ \\
\hline $2 \%$ Intralipid & 23.7 & 22.9 & $16-21^{\mathrm{c}}$ \\
\hline
\end{tabular}

a, From MIE theory (http://omlc.ogi.edu/software/mie/); b, from Vanstaveren et al. (1991) and Flock et al. (1992); c, values are estimated from values for $10 \%$ intralipid and empirical function from Zaccanti et al. (2003)

Table 1. Scattering coefficient values of several phantoms from the model Eq. (3) comparing with theoretical values or reported values (at $1300 \mathrm{~nm}$ ) and values from Faber model.

\subsubsection{Characterization of FMI and OCT signal}

A $400 \mu \mathrm{m}$ inner-diameter and $900 \mu \mathrm{m}$ outer-diameter capillary tube filled with different concentration of $\mathrm{Cy} 5.5$ was used as a phantom. It was placed in the scattering phantom containing $2 \%$ intralipid $\left(\mu_{\mathrm{s}} \sim 83 \mathrm{~cm}^{-1}\right.$ at FMI wavelengths, the scattering coefficient is close to skin scattering coefficient) (Troy \& Thennadil, 2001). The tube filled with fluorescence dye mimics a contrast-agent-labeled "tumor", while the intralipid overlaying at the top of "tumor" mimics the "skin" tissue.

In one set of experiments on the phantom, the center of the tube is set at $500 \mu \mathrm{m}$ beneath the surface and different concentration of Cy 5.5 was filled into the tube. FMI intensity was then measured for each configuration. Titration of the Cy5.5 concentration reveals a linear relationship between the FMI intensity and the dye concentration as shown in Figure 7(a).

In the second part of the experiment with the phantom, the relationship of the FMI intensity and the tube (dye) depth was studied by setting concentration of Cy 5.5 inside the capillary tube at $1 \mu \mathrm{M}$ and varying the tube depth (measured from the top surface of the tube) from 0 $\mu \mathrm{m}$ to $200 \mu \mathrm{m}$. The result, as presented in Figure 7(b), shows that the relationship can be well fitted into exponential function, which is a simplified model of Eq. (3) at small depths. It is consistent with other group's report (Turchin et al., 2005), which expects for small depths:

$$
\mathrm{I} \propto \exp \left(-2 \mu_{\mathrm{t}} \cdot \mathrm{d}\right) .
$$

where $\mu_{\mathrm{t}}=\mu_{\mathrm{s}}+\mu_{\mathrm{a}}$ is the total extinction coefficient. Both the absorption coefficient $\mu_{\mathrm{a}}$ of $10 \%$ intralipid and water are less than $0.01 \mathrm{~cm}^{-1}$ at FMI wavelengths (Flock et al., 1992; Pope \& Fry, 1997), while the scattering coefficient $\mu_{\mathrm{s}}$ at FMI wavelengths is $\sim 83 \mathrm{~cm}^{-1}, \sim 3-4$ orders of magnitude larger than the absorption coefficient. Therefore Eq. (9) can be simplified as:

$$
\mathrm{I} \propto \exp \left(-2 \mu_{\mathrm{s}} \cdot \mathrm{d}\right) .
$$

The scattering coefficient $\mu_{\mathrm{s}}$ can then be further determined from the fitting the measured data to Eq. (9b). From our measurement, $\mu_{\mathrm{s}}$ of $2 \%$ intralipid at $700 \mathrm{~nm}$ is about $88.0 \mathrm{~cm}^{-1}$, close to the reported value $83 \mathrm{~cm}^{-1}$ from the literature (Flock et al., 1992; Pope \& Fry, 1997). Although the model Eq. (3) works fine in fitting experimental data and finding the actual $\mu_{\mathrm{s}}$ values, it takes much longer time to fit than that using the simplified model Eq. (9b). If the medium is weakly scattering or only A-line profile near the surface are processed, the results from Eq. (3) and Eq. (9b) are close and we can use the simplified model Eq. (9b) to obtain $\mu_{\mathrm{s}}$ values. Figure 8 shows the differences between single scattering model Eq. (9b) and 
the model Eq. (3) for different $\mu_{\mathrm{s}}$ values and fitting depths. A simulated A-scan is first generated by Eq. (3) with a known $\mu_{\mathrm{s}}$ value, then the linear decay phase is fitted using Eq. (9b) to estimate the $\mu_{\mathrm{s}}$ value. Figure $8(\mathrm{~A})$ shows the fitted $\mu_{\mathrm{s}}$ values on the $120 \mu \mathrm{m}$ of a theoretical plot of A-scan profile based on Eq. (3). Figure 8(B) and 8(C) show that of $200 \mu \mathrm{m}$ and $280 \mu \mathrm{m}$ depths, correspondingly. In $120 \mu \mathrm{m}$ case, if the $\mu_{\mathrm{s}}$ value is between $15-165 \mathrm{~cm}^{-1}$,

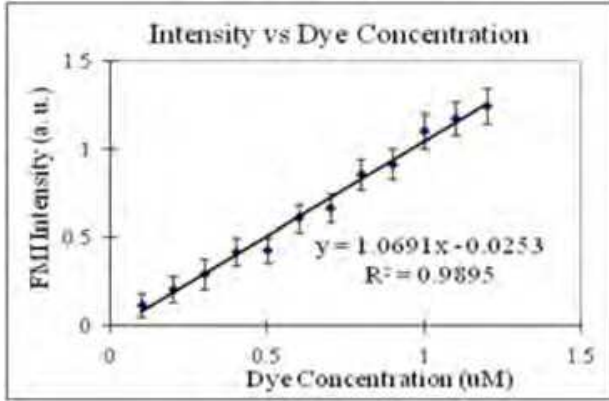

(a)

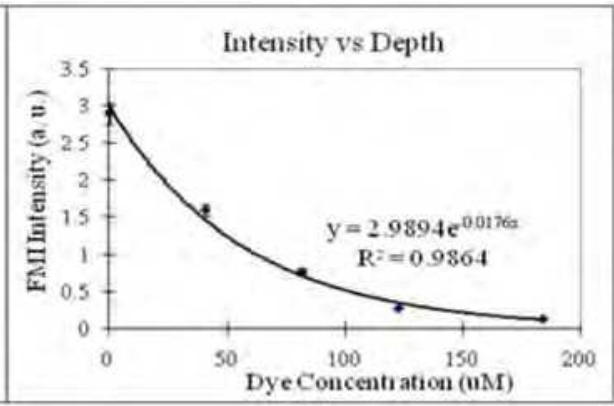

(b)

Fig. 7. (a) Relationship of fluorescence intensity versus Cy 5.5 concentration. A $400 \mu \mathrm{m}$ inner-diameter capillary tube filled with different concentration of Cy 5.5 was placed in 2\% intralipid (similar to skin scattering) and with the center at $500 \mu \mathrm{m}$ beneath the surface. (b) Relationship of fluorescence intensity versus capillary tube depth (depth of the top surface). The concentration of Cy 5.5 inside the tube was fixed at $1 \mu \mathrm{M}$. (Yuan et al., 2010b) with permission.
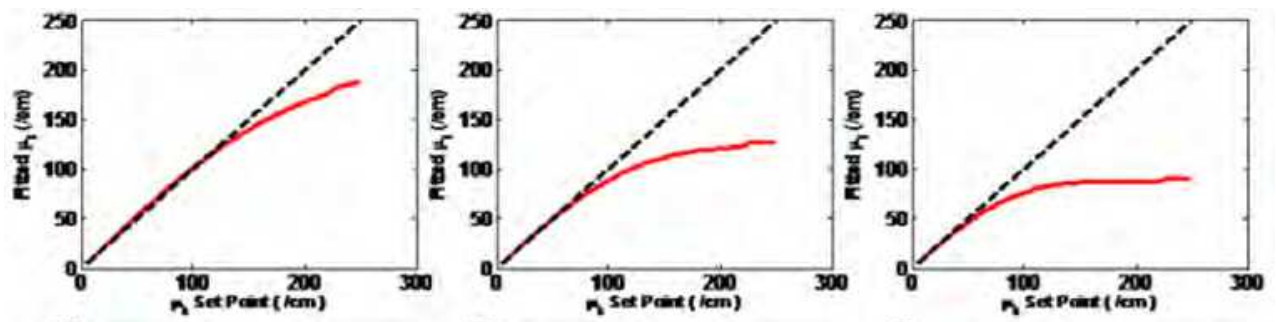

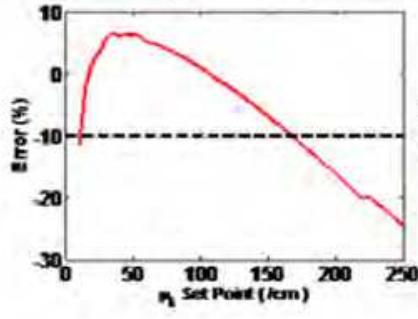

A

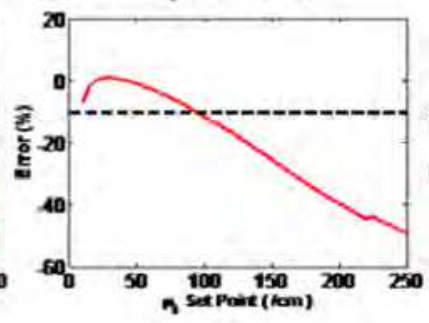

B

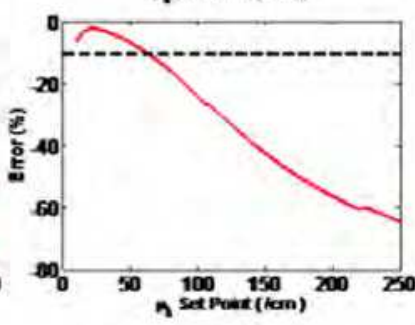

C

Fig. 8. The difference between the single scattering model from Eq. (9b) and the model from Eq. (3). (A) Fitted $\mu_{\mathrm{s}}$ values using single scattering model on theoretical plot of $120 \mu \mathrm{m} \mathrm{A-}$ scan profile from Eq. (3). (B) Fitted $\mu_{\mathrm{s}}$ values on theoretical plot of $200 \mu \mathrm{m}$ A-scan profile. (C) Fitted $\mu_{\mathrm{s}}$ values on theoretical plot of $280 \mu \mathrm{m}$ A-scan profile. The differences in percent between the two models are shown in the lower row. 
the difference between the fitting results from two models is less than $10 \%$. In $200 \mu \mathrm{m}$ case, if the actual $\mu_{\mathrm{s}}$ is between $10-90 \mathrm{~cm}^{-1}$, the difference is less than $10 \%$; for $280 \mu \mathrm{m}$ profile, 10 $60 \mathrm{~cm}^{-1} \mu_{\mathrm{s}}$ will result in a difference of less than $10 \%$.

\subsection{OCT imaging of mouse bowels}

Figure 9 shows the representative OCT images of both the normal and polypoid regions of mouse bowel. Figure 9(a) shows the cross-sectional OCT image of the normal bowel, with the characteristic villous and crypt structures. Figure $9(\mathrm{~b})$ shows the en face projection OCT image, which is analogous to the standard endoscope images. Individual villi/crypt can be visualized as well as the gross pattern of their distribution. Figure 9(c) shows the 3D reconstruction of the normal bowel. The OCT image Figure 9(a) agrees well with the histology shown in Figure 9(d).

In contrast, the polyp region shows the irregular mass in the OCT cross-sectional image in Figure 9(e). Projection OCT and 3D OCT in Figure 9(f) and 9(g) respectively show the whole polyp structures. Histology shown in Figure $9(\mathrm{~h})$ confirms the OCT imaging results. These results demonstrated OCT's ability to obtain micron-resolution, cross-sectional and 3D images of mouse bowel microstructures, and can be used to identify abnormalities such as the development of polyps.

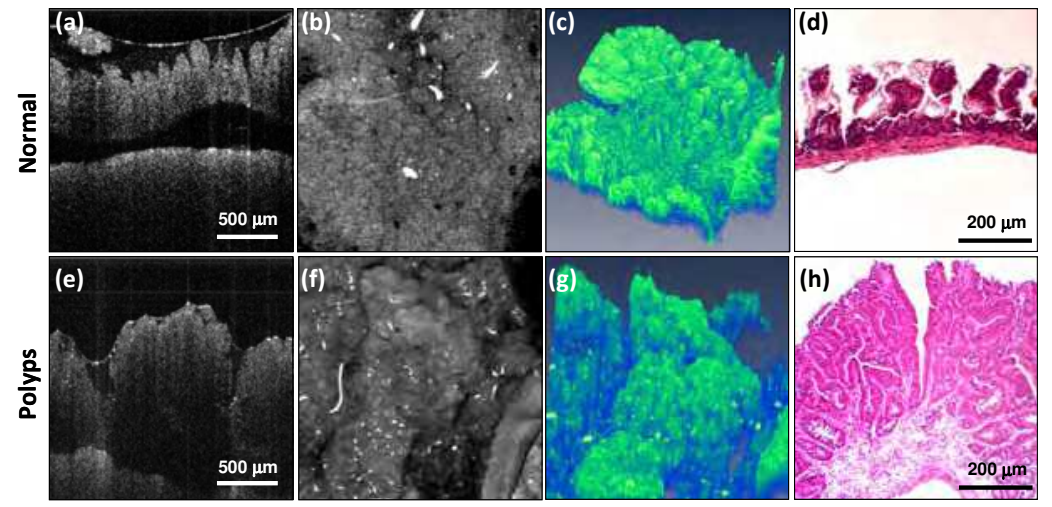

Fig. 9. OCT images of mouse bowel from two regions of interests (ROI), normal region and polypoid region. Normal region: (a) Cross-sectional OCT images. (b) OCT en face projection image. (c) 3-D OCT image (d) Histology. Polyps: (e) Cross-sectional OCT images. (f) OCT en face projection image. (g) 3-D OCT image (h) Histology. (Yuan et al., 2010b) with permission.

\subsection{OCT/FMI imaging of mouse bowels}

Figure 10 shows the results of the OCT/FMI imaging of mouse bowel polyp treated with UAE-1 conjugated liposome. Figure 10(A) is the height profile image obtained from OCT. The profile shows the height of the tissue surface estimated from OCT. Higher regions show red and lower regions show blue. Polyps regions usually rise up and are shown as red in the profile image. Figure 10(B) and 10(C) shows the corresponding cross-section OCT images of line 1 and 2 in Figure 10(A). Figure 10(D) is the scattering coefficient image. The scattering coefficient is obtained by fitting each OCT A-scan with Eq. (9b). 30 pixels ( $120 \mu \mathrm{m}$ in depth) beneath the tissue surface are used for each A-scan profile fitting. Simplified model 
Eq. (9b) is used because (1) the algorithm runs much faster than Eq. (3) and (2) $\mu_{\mathrm{s}}$ is about 60 $-150 \mathrm{~cm}^{-1}$ values and the difference of fitted $\mu_{\mathrm{s}}$ between two models are less than $10 \%$ as shown in Figure 8. Figure 10(E) shows the fluorescence image of the same ROI from FMI and Figure 10(F) is the fused image of Figure 10(D) and 10(E). Comparing Figure 10(A) and $10(\mathrm{~F})$, we could see that the polyp region shows a high scattering coefficient, possibly caused by the alteration of original structures. Fluorescence image further confirmed the polyp region, which shows high fluorescence signal around the edge. The binding mechanism of the UEA-1 contrast agent with polyps is still under investigation. Figure 10(F) shows very good correlation between OCT and FMI.

In conclusion, we have demonstrated a co-registered OCT and FMI imaging system. This system enables simultaneous imaging of tissue morphology and molecular information at high resolution over 2-3 mm field-of-view, and will have potential applications in small animal imaging and clinical imaging. Imaging results from our system on mouse intestinal tissues shows that glycoprotein targeting contrast agent promises to imaging polyps. This imaging technique is translatable to clinics in the forms of endoscopy, laparoscopy, and needle imaging probes, and might enable many biomedical applications.
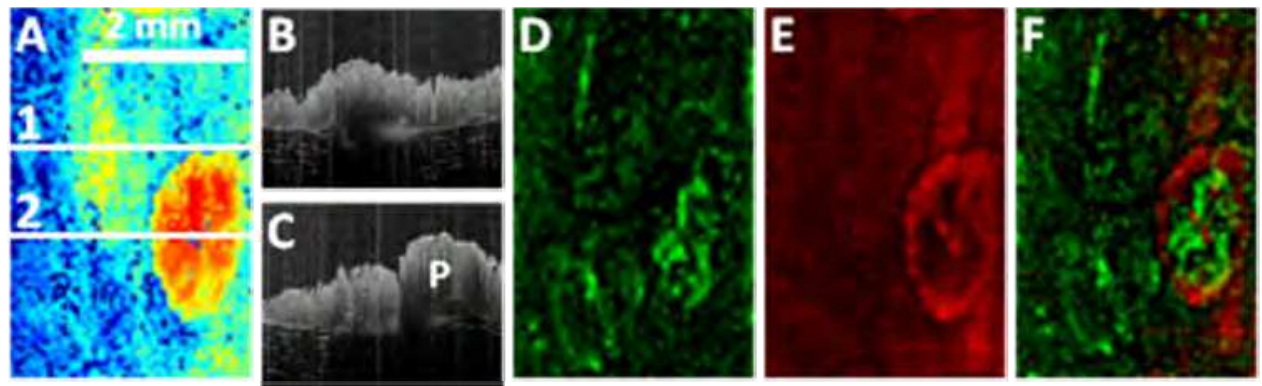

Fig. 10. OCT/FMI imaging of the polyp treated with UAE-1 conjugated liposome. (A) The height profile image obtained from OCT; $(B, C)$ The cross-section OCT images of line 1 ans 2 in Figure 9(A), correspondingly. (D) The scattering coefficient image in the same ROI obtained from OCT. The scale bar is [60150] $\mathrm{cm}^{-1}$. (E) The fluorescence image of the same ROI. (F) Fused image from D and E, showing very good co-registration. (Yuan et al., 2010a) with permission.

\section{Combining OCT with Fluorescence Laminar Optical Tomography (FLOT)}

\subsection{Introduction}

Although FMI technique works great for some applications, the depth-integrated fluorescence imaging has two limitations (Ntziachristos et al., 2002): 1) it cannot provide depth-resolved information, and 2) the detected signals non-uniformly depend on the depth. These present significant challenges in the quantitative investigation of the signal contributions. Diffuse optical tomography (DOT) has been successfully implemented in quantification of fluorescence contrast agents in tissues (Ntziachristos \& Weissleder, 2001; Li et al., 2003). But DOT works only for large volume tissues (cm and up). Laminar optical tomography (LOT) has been developed to perform depth-resolved functional imaging with $\sim 100 \mu \mathrm{m}$ resolution and up to $2.5 \mathrm{~mm}$ depth (Dunn \& Boas, 2000; Hillman et al., 2004). LOT 
is an extension of confocal microscopy using the multiple-displaced-confocal-pinhole design to capture the photons traveling through different depths, and the image is obtained through transport-based reconstruction (Dunn \& Boas, 2000). Fluorescence LOT (FLOT) has been demonstrated (Hillman et al., 2007). The comparable imaging scale (1-2 $\mathrm{mm}$ in depth) of OCT and FLOT as well as their complementary contrast mechanisms motivate the integration of OCT and FLOT as a multimodality imaging platform to provide tissue structural and molecular information in 3D in millimeter imaging scale.

A traditional FLOT system uses a point-focused light illumination and a 1D array detector to detect lights that undergo different depths. 3D images are obtained through 2D raster scanning of the illumination point. Alternatively, a line-field illumination can be utilized, and only $1 \mathrm{D}$ scanning is required to acquire 3D information. Line-scanning imaging has been successfully demonstrated in ophthalmoscopy (Iftimia et al., 2006), confocal microscopy (Dwyer et al., 2007), OCT (Yasuno et al., 2006), and optical coherence microscopy (Chen et al., 2007b). Line-scan imaging promises to alleviate the complicated 2D scanner design and therefore has strong potential to be miniaturized into endoscopic imaging devices. In addition, for LOT, line-scan imaging will use a 2D array detector such as a CCD camera as the detector. This may improve the imaging performance by using the high-pixel density CCD and will augment the widely available CCD-based fluorescence microscopes with the FLOT imaging mode. In this session, we will discuss combine OCT with line-scan FLOT.

\subsection{Methods}

\subsubsection{Fluorescence Laminar Optical Tomography (FLOT)}

Figure 11 shows the schematic of the co-registered OCT and line-scan FLOT imaging system. The left portion is the OCT subsystem, which is the same as the one used in OCT/FMI system. The line-scan FLOT system is shown in the right portion. The excitation source is a CW laser diode (Power Technology, Inc.) at $670 \mathrm{~nm}$. A cylindrical lens is used to expand the point illumination into a line. The excitation light is combined with the OCT sample arm by a dichroic mirror (DM-1). The reflectance and fluorescence light is separated by another dichroic mirror (DM-2), and the fluorescence signal is detected by an electronmultiplying CCD (EM-CCD) camera (Cooke Corp.) with an emission filter (700 $\pm 10 \mathrm{~nm}$ ). These wavelengths are chosen based on the optical properties of the near-IR dye Cy5.5. This system can be readily adapted to image other fluorescence dyes by changing the excitation source and emission filter wavelengths. The line illuminated on the sample measures 2.2 $\mathrm{mm}$ in length and $50 \mu \mathrm{m}$ in width with $8 \mathrm{~mW}$ power. The OCT and line-scan FLOT imaging are synchronized by scanning with the same galvanometer $(\mathrm{Y})$. The OCT beam is scanned in two axes (fast axis $X$ and slow axis $Y$ ). The scanning speed for $X$ is $25 \mathrm{~Hz}$ (with 624 samples in $2.5 \mathrm{~mm}$ ), and the scanning speed for $Y$ is $0.1 \mathrm{~Hz}$ (with 256 samples in $2.5 \mathrm{~mm}$ ). Line-scan FLOT scans in $Y$ only at the same speed as OCT, with 256 frames acquired by CCD (acquisition speed of $25 \mathrm{~Hz}$ with $30 \mathrm{~ms}$ exposure time). The resulting OCT 3D volume size is $624(X)$ by $256(Y)$ by $512(Z)$ pixels (with voxel size of $4 \mu \mathrm{m} \times 10 \mu \mathrm{m} \times 4.4 \mu \mathrm{m}$ ), and FLOT image raw data is $501(X)$ by $1024(Y)(2.2 \mathrm{~mm} \times 2.2 \mathrm{~mm})$ by $256(T)$ frames. The total acquisition time for each $3 \mathrm{D}$ volume is approximately $10 \mathrm{~s}$. The rate-limiting factors are the FLOT CCD frame rate, and the high number of OCT images acquired in the $Y$ dimension. The 3D imaging time can be significantly reduced by binning the CCD camera. 


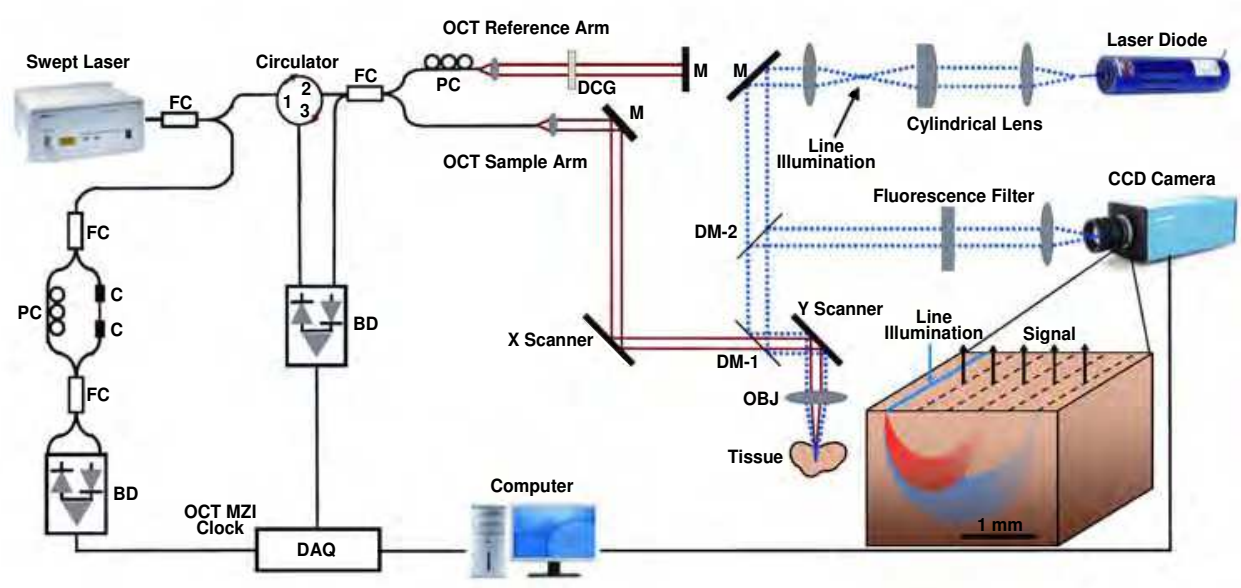

Fig. 11. Schematic of the combined OCT and line-scan FLOT system. (Chen et al., 2010) with permission.

\subsubsection{Data processing}

The depth-resolved FLOT images were obtained through an image reconstruction similar to those used in CT or DOT (Arridge, 1999). The fluorescence signal can be expressed as (Crilly et al., 1997; Hillman et al., 2007):

$$
F_{0}\left(\vec{r}_{d}, \widehat{\Omega}_{d}\right)=\frac{\sigma_{e x} \cdot \gamma}{4 \pi} \int \psi_{e x}\left(\vec{r}-\vec{r}_{s}\right) \cdot C_{f}(\vec{r}) \cdot \psi_{e m}\left(\vec{r}_{d}-\vec{r}\right) \cdot d^{3} \vec{r},
$$

where $\sigma_{\mathrm{ex}}$ is the absorption cross-section of the fluorophores at the excitation wavelength, $\gamma$ is the fluorescence quantum yield, $\Psi_{\text {ex }}\left(r-r_{s}\right)$ is the excitation fluence distribution calculated from the excitation photon radiance, $\mathrm{C}_{\mathrm{f}}(\mathrm{r})$ is the fluorophore concentration at position $\mathrm{r}$, and $\Psi_{\text {em }}\left(r_{d}-r\right)$ is the probability that a photon emitted by a source at position $r$ will be detected. Eq. (10) can be discretized into voxels, which yields a matrix equation:

$$
F=J C,
$$

where $\mathrm{F}$ is the fluorescence measurements, $\mathrm{C}$ is the spatially-distributed fluorophores concentration, and $\mathrm{J}$ is the weight function matrix that represent the sensitivity of each measurement to the fluorophore concentration at each voxel, and can be expressed as:

$$
J_{s, m}\left(\vec{r}_{s}, \vec{r}_{d}, \vec{r}\right)=D_{0} \cdot \psi_{e x}\left(\vec{r}-\vec{r}_{s}\right) \cdot \psi_{e m}\left(\vec{r}_{d}-\vec{r}\right),
$$

where $\mathrm{D}_{0}$ is a constant. Figure 12 shows the sensitivity distribution of FLOT using MonteCarlo simulation. The sensitivity profile varied as the source-detector separation $(\Delta)$ increased. Therefore, the depth-dependent information was encoded in the multiple detector meaurement, and was recovered through image reconstruction. The sensitivity matrix for line illumination was achieved by convolving the point-source sensitivity matrix along the $\mathrm{Y}$ dimension. 

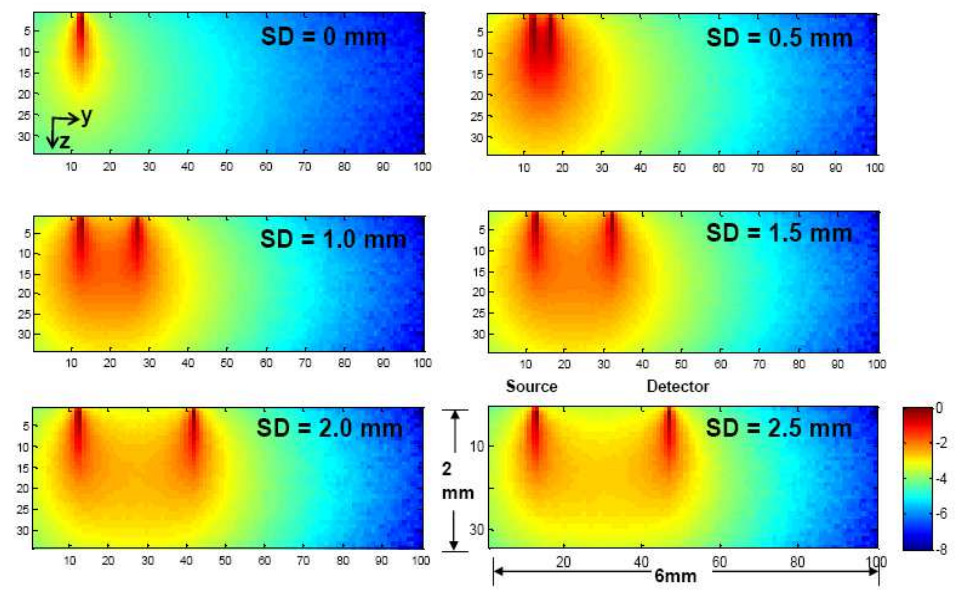

Fig. 12. Monte-Carlo simulated measurement sensitivity distribution of FLOT measurements (log scale). Tissue geometry is $3 \mathrm{~mm}$ (lateral) by $2 \mathrm{~mm}$ (depth) with scattering coefficient $\mu_{\mathrm{s}}=$ $8 \mathrm{~mm}^{-1}$ for excitation and $7 \mathrm{~mm}^{-1}$ for emission $(\mathrm{g}=0.9)$. (Chen et al., 2010) with permission.

\subsubsection{Imaging reconstruction in line-scan FLOT}

Image reconstruction is performed within individual planes $(Y Z)$ perpendicular to the illumination line $(X)$. To accelerate the reconstruction, the raw FLOT data are binned to 40 $(X)$ by $70(Y)$ pixels and downsampled to $64(T)$ frames. The corresponding pixel dimension is $55 \mu \mathrm{m} \times 30 \mu \mathrm{m}$ with a scan step of $40 \mu \mathrm{m}$. This is equivalent to FLOT measurements of 70 source-detector pairs with separations from 0 to $2.2 \mathrm{~mm}$. The selection of 70 source-detector pairs gives a sampling density of $30 \mu \mathrm{m}$ per detector interval, which provides sufficient sampling for the FLOT system. The cross-sectional FLOT image $(Y Z)$ is $70(Y) \times 60(Z)$ pixels with $30 \mu \mathrm{m} \times 30 \mu \mathrm{m}$ pixel dimensions. The reconstruction is based on the method discussed in session 4.2.2. The background optical properties for reconstruction are $\mu_{\mathrm{a}}=0.2 \mathrm{~mm}^{-1}$ and $\mu_{\mathrm{s}}$ $=7.2 \mathrm{~mm}^{-1}$ at $670 \mathrm{~nm}$ excitation, $\mu_{\mathrm{a}}=0.2 \mathrm{~mm}^{-1}$ and $\mu_{\mathrm{s}}=6.5 \mathrm{~mm}^{-1}$ at $700 \mathrm{~nm}$ emission, and $\mathrm{g}=$ 0.9. The image reconstruction is performed through the simultaneous iterative reconstruction technique approach (Kak et al., 1987), with iteration number $N=1000$ to make sure the change between iterations is $<0.1 \%$.

\subsection{FLOT phantom experiment}

We first experimentally checked the sensitivity distribution of FLOT calculated from MonteCarlo simulation by scanning the phantom: a capillary tube containing the fluorescent dye Cy5.5 using the FLOT system. Figure 13(A) shows a representative sensitivity matrix for line illumination and source-detection separation $\mathrm{d}=1.5 \mathrm{~mm}$. As the source detector scans through the object, the expected signal is shown in Figure 13(B), which is the profile at certain depth in the sensitivity matrix. Two peaks are present owing to the high sensitivity beneath either the source or the detector. Objects at different depths show different responses with more widely spread signals from the deeper object, owing to the stronger light scattering. The different profiles for objects with different depths contain the depth 
information and could be used to reconstruct the depth position of the object. Figures 13(C) shows the FLOT data for the capillary tube at different depths. The composite data is presented as a 2D matrix $(X T)$ of line profiles along the illumination axis $(X)$ at a specific position ( $Y=d$, where $d$ is the source-detector separation) with respect to the acquired frame time index ( $T$, scanning of the line illumination position). FLOT data for different sourcedetector separations $(0,0.5,1.0,1.5$ and $2.0 \mathrm{~mm})$ and object depths $(\sim 0$ and $\sim 0.8 \mathrm{~mm})$ are presented. The data show two lines with high intensities, which agrees with the simulation pattern in Figure 13(B). The object located at a deeper depth appears more blurred owing to scattering.
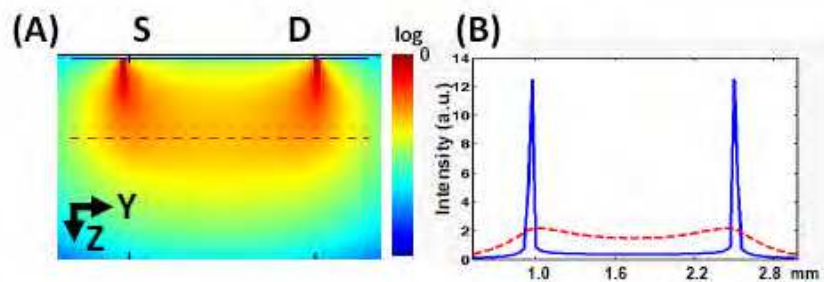

(C)

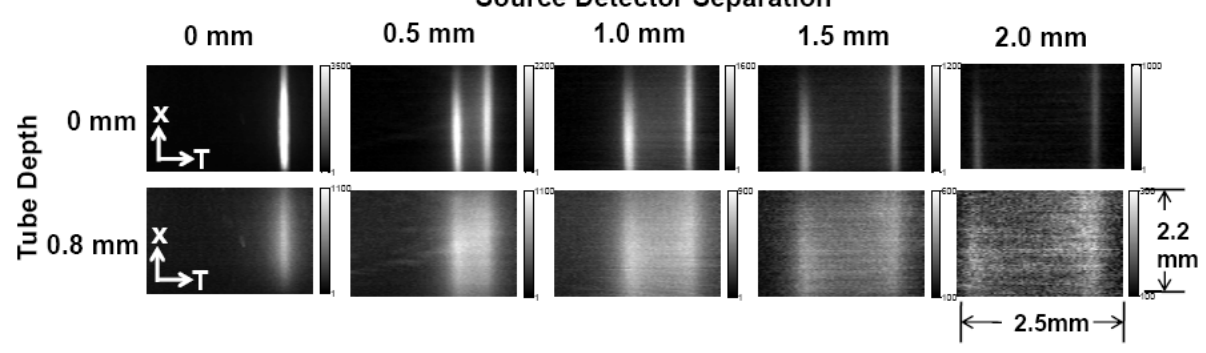

Fig. 13. (A) Monte Carlo simulated sensitivity distributions for line source and detector with separation $d=1.5 \mathrm{~mm}$. Log scale from -4 to 0 . (B) Intensity profiles for point object scanned through the $Y$ axis (solid curve, depth $\sim 0 \mathrm{~mm}$; dashed curve, $\sim 0.8 \mathrm{~mm}$ ). (C) FLOT measurement $(X T)$ for different source-detector separations from $Y=0 \mathrm{~mm}$ to $Y=2.0 \mathrm{~mm}$ ) for object depth $\sim 0 \mathrm{~mm}$ and $\sim 0.8 \mathrm{~mm}$. (Yuan et al., 2009) with permission.

To check the co-registration between OCT and FLOT, we obtained the depth-resolved 3D FLOT images through image reconstruction. Figure 14 shows the comparison of OCT and FLOT images of the capillary tube phantom. OCT readily images the 3D structure of the tube as shown in Figure 14(A). Figure 14(B) shows the comparison of OCT cross-sectional image (YZ) and FLOT image at location "1" denoted in (A). Cross-sectional OCT reveals the capillary tube (indicated by the arrow) with approximately $0.6 \mathrm{~mm}$ beneath the surface. FLOT cross-sectional image shows a fluorescence object at the same location, with slightly larger size due to the relatively larger FLOT resolution compared to OCT. Cross-sectional OCT and FLOT images at location "2" also show good agreement with the tube depth approximately $0.9 \mathrm{~mm}$ (Figure 14C). Compared to (B), the FLOT image for deeper object shows slightly enlargement in size. In addition, the peak values are slightly reduced when object gets deeper due to the enlargement of the reconstructed object size. Figure 14(D) 
shows the OCT cross-sectional image along the tube longitudinal dimension (XZ). The tube is placed with an angle with respect to the horizontal axis, and slightly bends flat. The FLOT image $(X Z)$ reveals similar contour of the capillary tube as shown by OCT. Also, this image is averaged over a range of $150 \mu \mathrm{m}$ in $\mathrm{Y}$ dimension along the center of the tube, and the results indicated nearly constant fluorophore concentration. In addition, we noted the decrease in the reconstructed fluorophore concentration $(3 \mu \mathrm{M}$ instead of $10 \mu \mathrm{M})$. This could be explained by the enlarged reconstructed object size $(\sim 300 \mu \mathrm{m}$ in diameter vs. $100 \mu \mathrm{m})$.

Here the OCT image provides the structural information of the phantom and the FLOT reconstructed image provides the fluorescence dye distribution information. In biomedical applications, FLOT would provide fluorescence-dye-targeted molecular information. So in biomedical applications, the hybrid system can be used for concurrent depth-resolved tissue-structural and molecular imaging.

In conclusion, we demonstrates the co-registered OCT and line-scanning FLOT system, which has strong potential to provide depth-resolved tissue structural and molecular information at millimeter imaging scale. This represents a new multimodal imaging regime and could be useful for investigating the tissue structure and function relationship. Future work will focus on the utilization of the OCT structural image as the a priori information to improve the FLOT reconstruction, and incorporation of line-scan OCT to co-register with line-scan FLOT for endoscopy imaging.
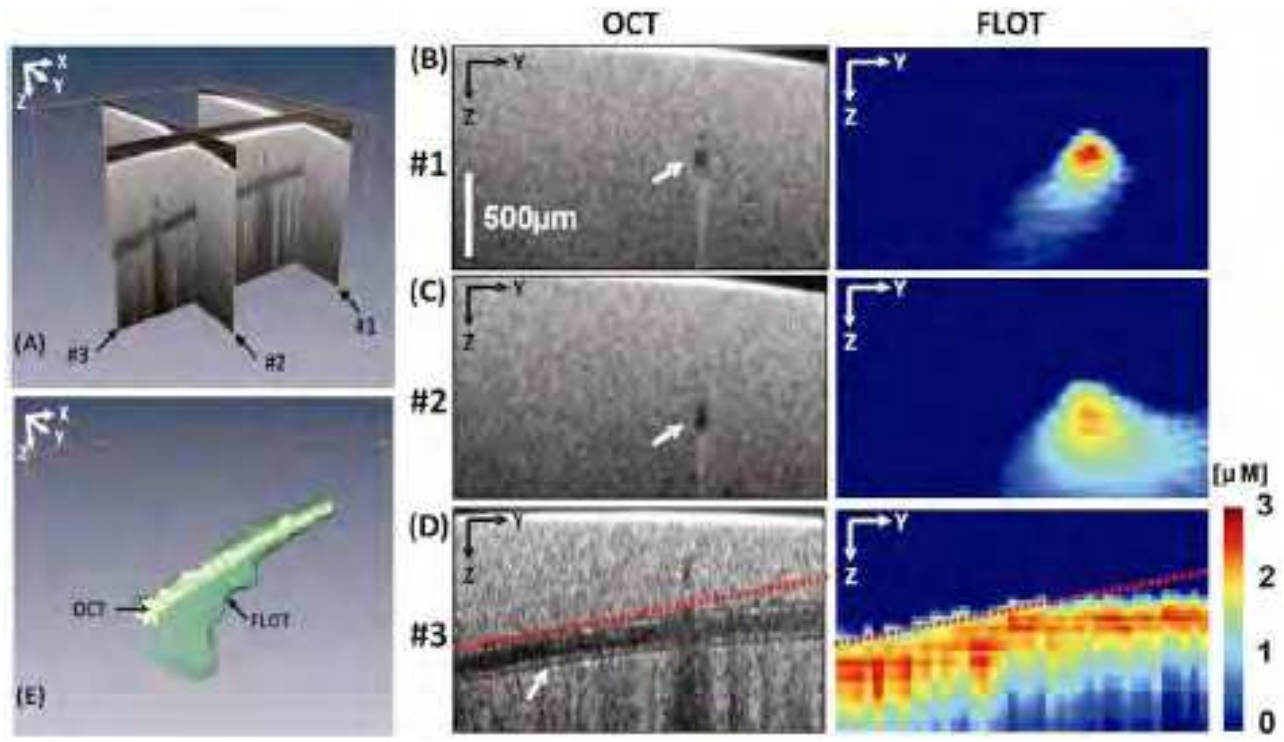

Fig. 14. Co-registration of OCT and line-scan FLOT of a capillary tube filled with fluorescence dye. (A) 3D OCT imaging showing three representative slices. (B) Left, OCT image (slice \#1) of the capillary tube (arrow); right, FLOT image reconstruction of the fluorescence object. (C) OCT and FLOT images of different position (slice \#2). (D) OCT and FLOT image (slice \#3) reveal the curvature of the capillary tube (two identical dotted lines serve as the reference slopes). (E) 3D isosurface of FLOT image shows good co-localization with OCT (Yuan et al., 2009) with permission. 


\section{Conclusion}

In this chapter, we introduce several multimodal optical imaging techniques which combines OCT and fluorescence imaging. OCT provides high-resolution, cross-sectional imaging of tissue microstructure, while fluorescence imaging reveals the biochemical and/or molecular information. Multimodal optical imaging techniques which combine OCT and fluorescence imaging could provide morphological, molecular and functional information simultaneously, and have great potential in disease diagnostics and therapy. Several such multimodal systems as well as their biomedical applications are introduced and discussed in this chapter. An ongoing development of novel multimodal system (OCT/FLOT) is also briefly introduced.

\section{Acknowledgement}

We thank Dr. David Boas (Massachusetts General Hospital) for providing the Monte-Carlo simulation code, Dr. Heng Lian (NanYang Technical University), Michael Lai (University of Maryland), and Qian Li (University of Maryland) for technical assistance of the imaging system and reconstruction algorithm, Drs. Ronald Summers and Celeste Roney (National Institutes of Health) for developing molecular imaging contrast agents, Dr. Ian White (University of Maryland) for the fabrication of capillary phantom, and Dr. James Jiang (Thorlabs, Inc.) for instrument support of SS-OCT. This research is supported by the UMCP GRB Research Award, Maryland Nano-Biotechnology Award, Minta-Martin Award, UMBUMCP SEED Grant Program, and the Prevent Cancer Foundation.

\section{References}

Achilefu, S. (2004) Lighting up tumors with receptor-specific optical molecular probes. Technology in Cancer Research \& Treatment, 3, 393-409.

Andrews, P. M., Chen, Y., Onozato, M. L., Huang, S. W., Adler, D. C., Huber, R. A., Jiang, J., Barry, S. E., Cable, A. E. \& Fujimoto, J. G. (2008) High-resolution optical coherence tomography imaging of the living kidney. Laboratory Investigation, 88, 441-449.

Arridge, S. R. (1999) Optical tomography in medical imaging. Inverse Problems, 15, R41R93.

Bouma, B. E., Tearney, G. J., Compton, C. C. \& Nishioka, N. S. (2000) High-resolution imaging of the human esophagus and stomach in vivo using optical coherence tomography. Gastrointestinal Endoscopy, 51, 467-474.

Brezinski, M. E., Tearney, G. J., Bouma, B. E., Izatt, J. A., Hee, M. R., Swanson, E. A., Southern, J. F. \& Fujimoto, J. G. (1996) Optical coherence tomography for optical biopsy. Properties and demonstration of vascular pathology. Circulation, 93, 12061213.

Chen, Y., Aguirre, A. D., Hsiung, P. L., Desai, S., Herz, P. R., Pedrosa, M., Huang, Q., Figueiredo, M., Huang, S. W., Koski, A., Schmitt, J. M., Fujimoto, J. G. \& Mashimo, H. (2007a) Ultrahigh resolution optical coherence tomography of Barrett's 
esophagus: preliminary descriptive clinical study correlating images with histology. Endoscopy, 39, 599-605.

Chen, Y., Huang, S. W., Aguirre, A. D. \& Fujimoto, J. G. (2007b) High-resolution linescanning optical coherence microscopy. Optics Letters, 32, 1971-1973.

Chen, Y., Yuan, S., Li, Q., Naphas, R., Wierwille, J., Blackwell, T. R., Winnard, P. T., Raman, V. \& Glunde, K. (2010) Integrated optical coherence tomography (OCT) and fluorescence laminar optical tomography (FLOT). IEEE Journal of Selected Topics in Quantum Electronics (In press).

Chinn, S. R., Swanson, E. A. \& Fujimoto, J. G. (1997) Optical coherence tomography using a frequency-tunable optical source. Optics Letters, 22, 340-342.

Crilly, R. J., Cheong, W. F., Wilson, B. \& Spears, J. R. (1997) Forward-adjoint fluorescence model: Monte Carlo integration and experimental validation. Applied Optics, 36, 6513-6519.

D'amico, A. V., Weinstein, M., Li, X., Richie, J. P. \& Fujimoto, J. (2000) Optical coherence tomography as a method for identifying benign and malignant microscopic structures in the prostate gland. Urology, 55, 783-787.

Danielson, B. L. \& Whittenberg, C. D. (1987) Guided-Wave Reflectometry with Micrometer Resolution. Applied Optics, 26, 2836-2842.

Dunn, A. \& Boas, D. (2000) Transport-based image reconstruction in turbid media with small source-detector separations. Optics Letters, 25, 1777-1779.

Dwyer, P. J., Dimarzio, C. A. \& Rajadhyaksha, M. (2007) Confocal theta line-scanning microscope for imaging human tissues. Applied Optics, 46, 1843-1851.

Faber, D., van der Meer, F., Aalders, M. \& van Leeuwen, T. (2004) Quantitative measurement of attenuation coefficients of weakly scattering media using optical coherence tomography. Optics Express, 12, 4353-4365.

Fercher, A. F., Drexler, W., Hitzenberger, C. K. \& Lasser, T. (2003) Optical coherence tomography-principles and applications. Reports on Progress in Physics, 66, 239303.

Fercher, A. F., Hitzenberger, C. K., Kamp, G. \& Elzaiat, S. Y. (1995) Measurement of Intraocular Distances by Backscattering Spectral Interferometry. Optics Communications, 117, 43-48.

Flock, S. T., Jacques, S. L., Wilson, B. C., Star, W. M. \& Vangemert, M. J. C. (1992) OpticalProperties of Intralipid - a Phantom Medium for Light-Propagation Studies. Lasers in Surgery and Medicine, 12, 510-519.

Fujimoto, J. G. (2003) Optical coherence tomography for ultrahigh resolution in vivo imaging. Nature Biotechnology, 21, 1361-1367.

Fujimoto, J. G., Brezinski, M. E., Tearney, G. J., Boppart, S. A., Bouma, B., Hee, M. R., Southern, J. F. \& Swanson, E. A. (1995) Optical biopsy and imaging using optical coherence tomography. Nature Medicine, 1, 970-972.

Fujimoto, J. G., Pitris, C., Boppart, S. A. \& Brezinski, M. E. (2000) Optical coherence tomography: an emerging technology for biomedical imaging and optical biopsy. Neoplasia, 2, 9-25. 
Haberland, U. H. P., Blazek, V. \& Schrnitt, H. J. (1998) Chirp optical coherence tomography of layered scattering media. Journal of Biomedical Optics, 3, 259-266.

Hariri, L. P., Tomlinson, A. R., Wade, N. H., Besselsen, D. G., Utzinger, U., Gerner, E. W. \& Barton, J. K. (2007) Ex vivo optical coherence tomography and laser-induced fluorescence spectroscopy imaging of murine gastrointestinal tract. Comparative Medicine, 57, 175-185.

Hariri, L. P., Tumlinson, A. R., Besselsen, D. G., Utzinger, U., Gerner, E. W. \& Barton, J. K. (2006) Endoscopic optical coherence tomography and laser-induced fluorescence spectroscopy in a murine colon cancer model. Lasers in Surgery and Medicine, 38, 305-313.

Hillman, E. M., Boas, D. A., Dale, A. M. \& Dunn, A. K. (2004) Laminar optical tomography: demonstration of millimeter-scale depth-resolved imaging in turbid media. Optics Letters, 29, 1650-1652.

Hillman, E. M. C., Bernus, O., Pease, E., Bouchard, M. B. \& Pertsov, A. (2007) Depthresolved optical imaging of transmural electrical propagation in perfused heart. Optics Express 15, 17827-17841.

Huang, D., Swanson, E. A., Lin, C. P., Schuman, J. S., Stinson, W. G., Chang, W., Hee, M. R., Flotte, T., Gregory, K., Puliafito, C. A. \& Fujimoto, J. G. (1991) Optical coherence tomography. Science, 254, 1178-1181.

Huber, R., Wojtkowski, M. \& Fujimoto, J. G. (2006) Fourier Domain Mode Locking (FDML): A new laser operating regime and applications for optical coherence tomography. Optics Express, 14, 3225-3237.

Iftimia, N. V., Hammer, D. X., Bigelow, C. E., Rosen, D. I., Ustun, T., Ferrante, A. A., Vu, D. \& Ferguson, R. D. (2006) Toward noninvasive measurement of blood hematocrit using spectral domain low coherence interferometry and retinal tracking. Optics Express, 14, 3377-3388.

Jang, I. K., Tearney, G. J., Macneill, B., Takano, M., Moselewski, F., Iftima, N., Shishkov, M., Houser, S., Aretz, H. T., Halpern, E. F. \& Bouma, B. (2005) In-vivo characterization of coronary atherosclerotic plaque by use of Optical Coherence Tomography. Circulation, 111, 1551-1555.

Jemal, A., Siegel, R., Ward, E., Hao, Y., Xu, J., Murray, T. \& Thun, M. J. (2008) Cancer statistics, 2008. CA a Cancer Journal for Clinicians, 58, 71-96.

Kak, A. C., Slaney, M. \& Ieee Engineering in Medicine and Biology Society. (1987) Principles of computerized tomographic imaging, New York, IEEE Press.

Levitz, D., Thrane, L., Frosz, M. H., Andersen, P. E., Andersen, C. B., Valanciunaite, J., Swartling, J., Andersson-Engels, S. \& Hansen, P. R. (2004) Determination of optical scattering properties of highly-scattering media in optical coherence tomography images. Optics Express, 12, 249-259.

Li, A., Miller, E. L., Kilmer, M. E., Brukilacchio, T. J., Chaves, T., Stott, J., Zhang, Q., Wu, T., Chorlton, M., Moore, R. H., Kopans, D. B. \& Boas, D. A. (2003) Tomographic optical breast imaging guided by three-dimensional mammography. Applied Optics, 42, 5181-5190. 
Li, X. D., Boppart, S. A., Van Dam, J., Mashimo, H., Mutinga, M., Drexler, W., Klein, M., Pitris, C., Krinsky, M. L., Brezinski, M. E. \& Fujimoto, J. G. (2000) Optical coherence tomography: advanced technology for the endoscopic imaging of Barrett's esophagus. Endoscopy, 32, 921-930.

Liu, B. \& Brezinski, M. E. (2007) Theoretical and practical considerations on detection performance of time domain, Fourier domain, and swept source optical coherence tomography. Journal of Biomedical Optics, 12, 044007.

Mandel, L. \& Wolf, E. (1995) Optical Coherence and Quantum Optics, Cambridge University Press, Cambridge, England.

Marten, K., Bremer, C., Khazaie, K., Sameni, M., Sloane, B., Tung, C. H. \& Weissleder, R. (2002) Detection of dysplastic intestinal adenomas using enzyme-sensing molecular beacons in mice. Gastroenterology, 122, 406-414.

Mcnally, J. B., Kirkpatrick, N. D., Hariri, L. P., Tumlinson, A. R., Besselsen, D. G., Gerner, E. W., Utzinger, U. \& Barton, J. K. (2006) Task-based imaging of colon cancer in the Apc(Min/+) mouse model. Applied Optics, 45, 3049-3062.

Ntziachristos, V., Tung, C. H., Bremer, C. \& Weissleder, R. (2002) Fluorescence molecular tomography resolves protease activity in vivo. Nature Medicine, 8, 757-760.

Ntziachristos, V. \& Weissleder, R. (2001) Experimental three-dimensional fluorescence reconstruction of diffuse media by use of a normalized Born approximation. Optics Letters, 26, 893-895.

Otis, L. L., Everett, M. J., Sathyam, U. S. \& Colston, B. W., Jr. (2000) Optical coherence tomography: a new imaging technology for dentistry. The Journal of the American Dental Association, 131, 511-4.

Pan, Y. T., Xie, T. Q., Du, C. W., Bastacky, S., Meyers, S. \& Zeidel, M. L. (2003) Enhancing early bladder cancer detection with fluorescence-guided endoscopic optical coherence tomography. Optics Letters, 28, 2485-2487.

Pope, R. M. \& Fry, E. S. (1997) Absorption spectrum (380-700 nm) of pure water. II. Integrating cavity measurements. Applied Optics, 36, 8710-8723.

Roney, C. A., Xie, J., Xu, B., Jabour, P., Griffiths, G. \& Summers, R. M. (2008) Glycoprotein expression by adenomatous polyps of the colon. Proceedings of SPIE, 6916, 691610.

Schmitt, J. M. (1999) Optical coherence tomography (OCT): a review. IEEE Journal of Selected Topics in Quantum Electronics, 5, 1205-1215.

Schmitt, J. M., Knuttel, A. \& Bonner, R. F. (1993) Measurement of Optical-Properties of Biological Tissues by Low-Coherence Reflectometry. Applied Optics, 32, 6032-6042.

Schuman, J. S., Puliafito, C. A. \& Fujimoto, J. G. (2004) Optical coherence tomography of ocular diseases (2nd Edition), Thorofare, NJ, Slack Inc.

Sivak, M. V., Jr., Kobayashi, K., Izatt, J. A., Rollins, A. M., Ung-Runyawee, R., Chak, A., Wong, R. C., Isenberg, G. A. \& Willis, J. (2000) High-resolution endoscopic imaging of the GI tract using optical coherence tomography. Gastrointestinal Endoscopy, 51, 474-479.

Tearney, G. J., Brezinski, M. E., Bouma, B. E., Boppart, S. A., Pitvis, C., Southern, J. F. \& Fujimoto, J. G. (1997) In vivo endoscopic optical biopsy with optical coherence tomography. Science, 276, 2037-2039. 
Troy, T. L. \& Thennadil, S. N. (2001) Optical properties of human skin in the near infrared wavelength range of 1000 to $2200 \mathrm{~nm}$. Journal of Biomedical Optics, 6, 167-176.

Tumlinson, A. R., Hariri, L. P., Utzinger, U. \& Barton, J. K. (2004) Miniature endoscope for simultaneous optical coherence tomography and laser-induced fluorescence measurement. Applied Optics, 43, 113-121.

Tung, C. H., Mahmood, U., Bredow, S. \& Weissleder, R. (2000) In vivo imaging of proteolytic enzyme activity using a novel molecular reporter. Cancer Research, 60, 4953-4958.

Turchin, I. V., Sergeeva, E. A., Dolin, L. S., Kamensky, V. A., Shakhova, N. M. \& RichardsKortum, R. (2005) Novel algorithm of processing optical coherence tomography images for differentiation of biological tissue pathologies. Journal of Biomedical Optics, 10, 064024.

Vanstaveren, H. J., Moes, C. J. M., Vanmarle, J., Prahl, S. A. \& Vangemert, M. J. C. (1991) Light-Scattering in Intralipid-10-Percent in the Wavelength Range of 400-1100 nm. Applied Optics, 30, 4507-4514.

Wang, C. S., Cheng, W. H., Hwang, C. J., Burns, W. K. \& Moeller, R. P. (1982) High-Power Low-Divergence Super-Radiance Diode. Applied Physics Letters, 41, 587-589.

Wang, R. K. (2007) Fourier domain optical coherence tomography achieves full range complex imaging in vivo by introducing a carrier frequency during scanning. Physics in Medicine and Biology, 52, 5897-5907.

Wang, Z. G., Durand, D. B., Schoenberg, M. \& Pan, Y. T. (2005) Fluorescence guided optical coherence tomography for the diagnosis of early bladder cancer in a rat model. Journal of Urology, 174, 2376-2381.

Welzel, J., Reinhardt, C., Lankenau, E., Winter, C. \& Wolff, H. H. (2004) Changes in function and morphology of normal human skin: evaluation using optical coherence tomography. British Journal of Dermatology, 150, 220-225.

Wojtkowski, M., Leitgeb, R., Kowalczyk, A., Bajraszewski, T. \& Fercher, A. F. (2002) In vivo human retinal imaging by Fourier domain optical coherence tomography. Journal of Biomedical Optics, 7, 457-463.

Yasuno, Y., Endo, T., Makita, S., Aoki, G., Itoh, M. \& Yatagai, T. (2006) Three-dimensional line-field Fourier domain optical coherence tomography for in vivo dermatological investigation. Journal of Biomedical Optics, 11, 014014.

Youngquist, R., Carr, S. \& Davies, D. (1987) Optical coherence-domain reflectometry: a new optical evaluation technique. Optics Letters, 12, 158-160.

Yuan, S., Li, Q., Jiang, J., Cable, A., \& Chen, Y. (2009) Three-dimensional coregistered optical coherence tomography and line-scanning fluorescence laminar optical tomography. Optics Letters, 34, 1615-1617.

Yuan,S., Roney, C. A., Li, Q., Jiang, J., Cable, A., Summers, R. M. \& Chen, Y. (2010a) Correlation of morphological and molecular parameters for colon cancer. Proceedings of SPIE 7555. (Submitted)

Yuan,S., Roney, C. A., Wierwille, J., Chen, C. W., Xu, B., Griffiths, G., Jiang, J., Ma, H., Cable, A., Summers, R. M. \& Chen, Y. (2010b) Co-registered optical coherence tomography 
and fluorescence molecular imaging for simultaneous morphological and molecular imaging. Physics in Medicine and Biology, 55, 191-206.

Zaccanti, G., Del Bianco, S. \& Martelli, F. (2003) Measurements of optical properties of highdensity media. Applied Optics, 42, 4023-4030. 


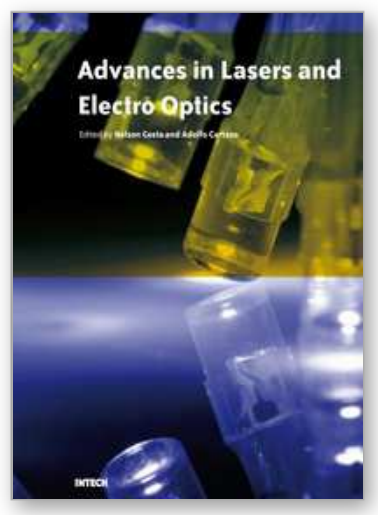

\author{
Advances in Lasers and Electro Optics \\ Edited by Nelson Costa and Adolfo Cartaxo
}

ISBN 978-953-307-088-9

Hard cover, 838 pages

Publisher InTech

Published online 01, April, 2010

Published in print edition April, 2010

Lasers and electro-optics is a field of research leading to constant breakthroughs. Indeed, tremendous advances have occurred in optical components and systems since the invention of laser in the late $50 \mathrm{~s}$, with applications in almost every imaginable field of science including control, astronomy, medicine, communications, measurements, etc. If we focus on lasers, for example, we find applications in quite different areas. We find lasers, for instance, in industry, emitting power level of several tens of kilowatts for welding and cutting; in medical applications, emitting power levels from few milliwatt to tens of Watt for various types of surgeries; and in optical fibre telecommunication systems, emitting power levels of the order of one milliwatt. This book is divided in four sections. The book presents several physical effects and properties of materials used in lasers and electro-optics in the first chapter and, in the three remaining chapters, applications of lasers and electro-optics in three different areas are presented.

\title{
How to reference
}

In order to correctly reference this scholarly work, feel free to copy and paste the following:

Shuai Yuan and Yu Chen (2010). Combining Optical Coherence Tomography with Fluorescence Imaging, Advances in Lasers and Electro Optics, Nelson Costa and Adolfo Cartaxo (Ed.), ISBN: 978-953-307-088-9, InTech, Available from: http://www.intechopen.com/books/advances-in-lasers-and-electro-optics/combiningoptical-coherence-tomography-with-fluorescence-imaging

\section{INTECH}

open science | open minds

\section{InTech Europe}

University Campus STeP Ri

Slavka Krautzeka 83/A

51000 Rijeka, Croatia

Phone: +385 (51) 770447

Fax: +385 (51) 686166

www.intechopen.com

\section{InTech China}

Unit 405, Office Block, Hotel Equatorial Shanghai

No.65, Yan An Road (West), Shanghai, 200040, China

中国上海市延安西路65号上海国际贵都大饭店办公楼 405 单元

Phone: +86-21-62489820

Fax: $+86-21-62489821$ 
(C) 2010 The Author(s). Licensee IntechOpen. This chapter is distributed under the terms of the Creative Commons Attribution-NonCommercialShareAlike-3.0 License, which permits use, distribution and reproduction for non-commercial purposes, provided the original is properly cited and derivative works building on this content are distributed under the same license. 